\title{
Research on the Relationship between Corporate Social Responsibility Characteristics and Corporate Double Reputation
}

\author{
- Based on the adjustment effect of social media capabilities \\ Xiaochun $\mathrm{Ge}^{1, \mathrm{a}}$,Mengqi Zhao ${ }^{2, \mathrm{~b}}$ \\ 'Zhejiang Gongshang University, Hangzhou 310000, China; \\ 2Zhejiang Gongshang University, Hangzhou 310000, China. \\ age19780@163.com, b1273465225@qq.com
}

Keywords: Corporate social responsibility characteristics, Cognitive reputation, Emotional reputation, Social media capabilities.

\begin{abstract}
In this paper, 294 questionnaires were used to explore the influence of corporate social media capability on the relationship between corporate social responsibility characteristics and corporate double reputation. The empirical analysis was carried out by SPSS multiple regression statistical method. The results are as follows: (1) The characteristics of corporate social responsibility (commitment, level, time choice, degree of relevance) positively affect the cognitive reputation and emotional reputation of the enterprise; (2) The cognitive reputation of the company positively affects the emotional reputation; (3) The social media capability of enterprises positively adjusts the relationship between CSR characteristics (commitment, level) and cognitive reputation; (4) The social media capability of enterprises positively adjusts the relationship between CSR characteristics (commitment, level and time choice) and emotional reputation.

\section{企业社会责任特征与企业双重声誉关系研究 \\ 一一基于社交媒体能力的调节效应 \\ 葛笑春 $1, \mathrm{a}$,赵孟齐 $2, \mathrm{~b}$ \\ 1 浙江工商大学, 杭州 310000 , 中国; \\ 2 浙江工商大学, 杭州 310000 , 中国; age19780@163.com, b1273465225@qq.com}

关键词: 企业社会责任特征; 认知声誉; 情感声誉; 社交媒体能力

摘要：本文以 294 份问卷探讨了企业社交媒体能力对企业社会责任特征与 企业双重声誉关系的影响。运用 SPSS 多元回归统计的方法进行实证分析。结 果：（1）企业社会责任特征（承诺、水平、时间选择、关联度）分别正向影响 企业的认知声誉和情感声誉;（2）企业的认知声誉正向影响情感声誉; 企业的社交媒体能力正向调节企业社会责任特征（承诺、水平）和认知声誉的 关系；（4）企业的社交媒体能力正向调节企业社会责任特征（承诺、水平和时 间选择）和情感声誉的关系。

作者简介：葛笑春 $(1978-)$ ，女（汉)，辽宁丹东人，浙江工商大学工商管理学院副教授，博士，硕士生导师，研究方向 为跨部门联盟、企业社会责任等。赵孟齐 (1994-), 女 (汉), 河南安阳人, 浙江工商大学工商管理学院硕士研究生, 研究方 向为企业社会责任、非盈利组织向社会企业转型等。

基金项目：教育部青年基金课题 16YJC630088；浙江省高校人文社科重点研究基地课题 JYTgs20151103 


\section{一、引言}

市场经济的快速发展促进企业间日益激烈的竞争, 要想在激烈的市场竞争中获得可持续 的竞争优势, 就要把战略的眼光从有形资产转向无形资产。近年来, 国内外学者对无形资产 的研究聚焦于企业声誉, 并认为企业声誉作为一种可以成为竞争优势来源的无形资产, 是组 织过去行为和利益相关者头脑中未来前景的稳定总体感知表示, 可以某种标准衡量 $[1,2]$ 。 纵观国内外权威的企业声誉评价体系, 企业社会责任都是其中的一个重要指标。如《财富》 杂志的全美（球）最受欢迎的公司 AMAC（GMAC）评选的九大指标包括了 “社区和环境责 任”; 英国《当代管理》杂志的英国最受尊敬的企业评选的指标中也包括了 “社区和环境责 任”; 德国《管理者杂志》的 “综合商誉” 评价指标包括了环境责任; 我国《经济观察报》 的中国最受尊敬的企业评选中包括了 “社会责任感和工作环境” 等。企业社会责任的重要性 已经得到各界的公认, 企业也在社会责任方面投入越来越多的资源, 要有效, 企业社会责任 信息必须传递给利益相关者。互联网时代社交媒体的兴起为企业社会责任的沟通带来了极大 的便利, 企业可以利用外部 (如 Twitter) 和内部社交媒体来学习和吸收市场知识 $[3,4]$, 管理与潜在和当前客户以及其他利益相关者的关系 $[5,6]$, 并提高他们的在线企业声誉 $[7]$ 。

然而, 企业社会责任对组织结果 (如企业声誉和财务绩效) 影响的研究一直没有定论 [8], 企业社会责任对企业声誉的影响可能因行业、地域、利益相关者的不同而有所差别 [9]。此外, 研究社交媒体与 CSR 活动（即一种商业活动） [10,11] 之间的关系的论文很少, 也少有研究进一步阐述这两个概念之间的关系以及它们对企业声誉的联合效应 [12]。

基于此, 本文从互联网时代企业社交媒体能力切入, 通过实证研究检验企业社会责任和 企业声誉之间的关系，并分析社交媒体能力对这一关系的影响。

\section{二、文献综述与研究假设}

1. 企业社会责任和企业声誉

近年来, 国内外学者都热衷于企业社会责任和企业声誉关系的研究。本文在对前辈们研 究成果梳理的基础上发现: 大多数研究证实, 企业社会责任对企业声誉有着正向的促进作 用。本文对现有研究的梳理如下:

第一，整体角度的研究。Fornbrun and Shanley[13]最早提出公司履行企业社会责任对 企业声誉的构建具有积极影响。Brammer and Pavelin[14]通过对 227 家英国上市公司的研 究得出通过正确确定企业社会责任战略的适用范围可以帮助企业声誉构建的结论。Lai [15] 指出企业承担社会责任可以增强消费者对企业声誉的感知，进而提升企业的品牌形象。 Maden [16] 研究 CSR 对企业声誉的影响, 结果证明 CSR 不仅是企业声誉的前置变量, 并且有 强的正向影响。Elisa[17]通过对西班牙上市公司的研究得出结论, 不仅企业社会责任的履 行对企业声誉有着正向的影响，而且透明度在两者的关系中发挥着中介作用。

第二，不同利益相关者角度的研究。从利益相关者的不同角度出发，沈泽 [18]基于消费 者视角, 研究三个不同行业的企业社会责任对企业声誉的影响, 结果表明企业的产品服务责 任、社区支持责任及环境保护责任会对企业的认知声誉和情感声誉产生显著的影响, 且不同 行业间存在着差异。李海芹 [19]从消费者角度研究企业社会责任的不同维度（经济责任、环 境责任、消费者责任、员工责任、法律责任、慈善责任）对企业认知声誉和情感声誉的影 响, 除了环境责任和对员工的责任对企业声誉的两个维度都没有产生显著影响, 其他维度都 对企业的认知声誉和情感声誉产生不同程度的影响, 且认知声誉对情感声誉也有着正向影 响。田虹 [20]将企业社会责任分为企业社区责任和企业环境责任, 分别探讨其对企业声誉的 作用机制, 分析利益相关者压力和道德滑坡对二者关系的调节效应, 对企业社会责任履行的机 理进行系统性论证。研究结果表明, 企业社区责任和企业环境责任对企业声誉具有正向的推 动作用。

基于此, 本研究提出初始命题: 企业社会责任正向影响企业声誉。

企业社会责任与企业声誉的关系一直受到学术界和企业界的关注。尽管已有大量学者采 用不同的衡量指标、测量方法甚至开发更新更完备的评估模型，也基于不同的利益相关者的 
角度对两者的关系展开研究, 大多数学者得出的结论是企业社会责任正向影响企业的声誉。 太过一致化的研究结论也凸显了两个问题: 一是这些研究虽有助于深入理解企业社会责任, 但大多数集中在企业社会责任的内容、过程或结果上，在实践中易导致企业社会责任报告日 益同质化及几近标准化, 只简单记录与企业社会责任相关的活动而不深入理解其前因不大可 能揭示企业间的真实区别, 因为这忽略了企业从事社会责任活动的内在本质一一企业社会责 任特征。本研究借鉴刘风军等 [21] 的研究, 将企业社会责任特征分为企业社会责任承诺、企 业社会责任水平、企业社会责任关联度和企业社会责任时间选择四个维度展开研究; 二是部 分研究将企业声誉作为一个整体的变量且衡量标准也较为简单, 而这并不能帮助企业更好的 对声誉进行管理。因此, 本文遵循 Manfred[22]提出的企业声誉的二维模型, 即声誉是一个 由情感和认知两个部分组成的结构, 并从两个维度对声誉展开研究。

因此，提出假设：

H1：企业社会责任特征正向影响企业认知声誉。

$\mathrm{H} 1 \mathrm{a}$ ：企业社会责任承诺正向影响企业认知声誉;

$\mathrm{H} 1 \mathrm{~b}$ ：企业社会责任水平正向影响企业认知声誉;

H1c：企业社会责任时间选择正向影响企业认知声誉;

H1d: 企业社会责任关联度正向影响企业认知声誉;

H2：企业社会责任特征正向影响企业情感声誉。

H2a：企业社会责任承诺正向影响企业情感声誉;

$\mathrm{H} 2 \mathrm{~b}$ ：企业社会责任水平正向影响企业情感声誉;

H2c：企业社会责任时间选择正向影响企业情感声誉;

$\mathrm{H} 2 \mathrm{~d}$ ：企业社会责任关联度正向影响企业情感声誉;

2. 企业认知声誉对情感声誉的影响

如上所述, 对企业声誉进行评价时要从认知和情感两个维度展开。从心理学角度而言, 认知和情感是两个独立的心里层面, 但关于 “认知和情感谁先产生” 这一遗留问题至今仍未 得出一致的结论 [19]。就本研究而言, 利益相关者要对企业的声誉进行评价, 首先要对企业 的业务范围、经营能力、企业社会责任活动等有一个客观的认识, 即产生认知声誉评价。而 后在认知基础上产生情感。因此, 提出假设 :

H3：企业认知声誉对企业情感声誉有正向影响。

3. 企业社交媒体能力对企业社会责任和企业声誉关系的影响

社交媒体能力是指企业利用社交媒体 (如 Facebook, Twitter and corporate blogs) 执行公司业务活动的能力 $[23,24]$ 。企业通过社交媒体进行企业社会责任活动的有效性尚不 明确。一些研究表明, 通过社交媒体平台传达的 CSR 信息对追随者的影响最小, 因为他们只 能阅读社交媒体中散布的信息的一小部分 [25]。然而, 另外一些学者则认为, 社交媒体是分 散企业社会责任活动, 影响利益相关者行为和态度的一个关键工具, 也是企业信息可信度和 利益相关者对企业认同感提高的有效途径 [26]。

本研究认为, 社交媒体能力可能在 CSR 活动与企业声誉之间起到放大作用, 即在社交媒 体能力的存在下, CSR 活动与企业声誉之间的关系可能更强。这个命题可以通过几个论点来 解释。企业社会责任活动和社交媒体能力是两个相互加强的资源/能力 [27]。企业对社交媒 体的使用, 使企业利益相关者能够更多地了解企业社会责任活动, 从而建立更强大的企业品 牌, 从而最大限度地提高企业社会责任活动的商业价值。整体 (CSR 活动*社交媒体能力) 的效用不仅仅是其部分（CSR 活动和社交媒体能力）的总和。因此，提出假设：

H4：企业社交媒体能力正向调节企业社会责任特征与企业认知声誉的关系。

$\mathrm{H} 4 \mathrm{a}$ : 企业社交媒体能力正向调节企业社会责任承诺与企业认知声誉的关系;

H4b：企业社交媒体能力正向调节企业社会责任水平与企业认知声誉的关系;

H4c：企业社交媒体能力正向调节企业社会责任时间选择与企业认知声誉的关系;

H4d：企业社交媒体能力正向调节企业社会责任关联度与企业认知声誉的关系; 
H5：企业社交媒体能力正向调节企业社会责任特征与企业情感声誉的关系。

$\mathrm{H} 5 \mathrm{a}$ ：企业社交媒体能力正向调节企业社会责任承诺与企业情感声誉的关系;

H5b：企业社交媒体能力正向调节企业社会责任水平与企业情感声誉的关系;

H5c：企业社交媒体能力正向调节企业社会责任时间选择与企业情感声誉的关系;

$\mathrm{H} 5 \mathrm{~d}$ ：企业社交媒体能力正向调节企业社会责任关联度与企业情感声誉的关系;

在理论文献研究的基础上, 根据以上假设, 我们提出 “企业社会责任特征与企业双重声 誉关系研究一一基于社交媒体能力的调节效应” 的概念模型（见图 1)。

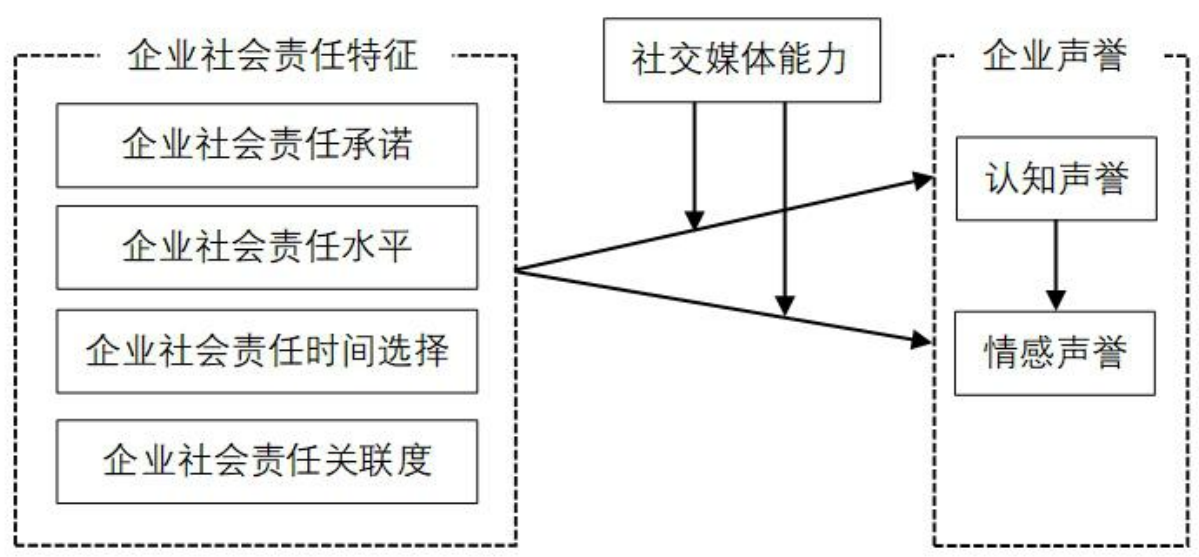

图 1 社交媒体能力视角下企业社会责任特征对企业双重声誉的影响机理研究

\section{三、研究设计}

1. 研究样本

本研究调查对象为浙江省内各地市企业高层、中层和基层管理者, 调查问卷主要通过线 下 MBA 课堂和线上问卷星两个渠道发放。本次共发放 382 份，回收 335 份，回收率为 87. 70\%; 其中有效问卷 294 份（包括纸质版 145 份，问卷星 149 份）。有效样本分布情况 为: 高层管理者占 $5.10 \%$, 中层管理者占 $22.45 \%$, 基层管理者占 $72.45 \% ; 100$ 人以下企业占 $12.93 \% ， 101-500$ 人企业占 $20.75 \% ， 501-1000$ 人企业占 $17.68 \% ， 1000$ 人以上企业占 48. $64 \%$; 孕育期企业占 $2.38 \%$, 发展期企业占 $18.71 \%$, 成长期企业占 $26.87 \%$, 成熟期企业 占 48.64\%，衰退期企业占 3.40\%；国企占 $22.45 \%$ ，民营企业占 $62.59 \%$ ，外资企业占 $7.14 \%$, 其它占 $7.82 \%$; 上市企业占 30.95\%, 非上市企业占 $69.05 \%$; 企业所属行业以制造和 金融业为主，分别占 $28.57 \%$ 和 $17.69 \%$ ，其它行业占 $53.74 \%$ 。

\section{2. 变量测量}

企业社会责任特征借鉴刘风军等 [21]的研究, 将企业社会责任特征分为企业社会责任承 诺、企业社会责任水平、企业社会责任关联度和企业社会责任时间选择四个维度, 具体为企 业社会责任承诺 8 个题项，企业社会责任水平 8 个题项，企业社会责任时间选择 7 个题项， 企业社会责任关联度 4 个题项。企业声誉遵循 Manfred[22]和刘郬 [28]提出的二维模型，即 声誉是由情感和认知两个部分组成的。在声誉的测量上, 借鉴刘㙐 [28]提出的企业声誉测量 指标, 具体为认知声誉 5 个题项, 情感声誉 7 个题项。而关于企业社交媒体能力的测量则是 借鉴Braojos [24] 的研究成果, 共 5 个题项。所有量表均采用 7 级李克特计分法, 1-7 分别 从“完全不同意” 到 “完全同意”。

为有效避免研究结论的偏差, 选取了职位等级、企业员工人数、企业所处生命周期阶 段、企业性质、是否上市和企业所属行业等 6 个控制变量。

\section{3. 研究结果}

3.1 各量表的信效度分析

使用 SPSS20. 0 对所有量表进行信效度分析, 结果如表 1 所示, 7 个量表的 Cronbach’ s Alpha 系数均在 0.70 以上, 说明各量表具有内部一致性。且所有量表的 KMO 值均大于 0.7 , Bartlett 球形检验结果均显著, 累计解释方差均大于 $50 \%$, 通过效度检验。说明回收问卷所 
获得的数据具有较高可信度。

表 1 各量表的信效度分析结果 $(\mathrm{N}=294)$

\begin{tabular}{cccc}
\hline 变量 & Cronbach’ s Alpha 系数 & KM0 值 & 累计解释方差 \\
\hline 企业社会责任承诺 & 0.924 & 0.913 & $66.765 \%$ \\
企业社会责任水平 & 0.911 & 0.917 & $61.782 \%$ \\
企业社会责任时间选择 & 0.925 & 0.904 & $69.204 \%$ \\
企业社会责任关联度 & 0.837 & 0.796 & $67.593 \%$ \\
认知声誉 & 0.935 & 0.886 & $79.594 \%$ \\
情感声誉 & 0.968 & 0.945 & $84.039 \%$ \\
社交媒体能力 & 0.808 & 0.784 & $56.989 \%$ \\
\hline
\end{tabular}

3.2 描述性统计及相关分析

所有变量的均值、标准差和相关系数矩阵见表 2 。可以看出企业社会责任特征的四个维 度与认知声誉和情感声誉均为正相关, 初步验证了第二部分所提出的假设 (H1 和 H2), 可以 进行下一步的回归模型检验。且 RZSY 对 QGSY 有显著正向影响 ( $\beta=0.824, \quad \mathrm{p}<0.01)$, 假设 H3 成立。

表 2 研究变量的平均数、标准差和相关分析结果 (N=294)

\begin{tabular}{llllllllll}
\hline 变量 & 均值 & 标准差 & 1 & 2 & 3 & 4 & 5 & 6 & 7 \\
\hline CN & 5.3929 & 1.05765 & & & & & & & \\
SP & 5.0664 & 1.03216 & $.816^{* *}$ & & & & & \\
SJ & 5.2859 & 1.09194 & $.818^{* *}$ & $.895^{* *}$ & & & & & \\
GLD & 4.9915 & 1.01731 & $.651^{* *}$ & $.777^{* *}$ & $.805^{* *}$ & & & & \\
RZSY & 5.1476 & 1.25922 & $.769^{* *}$ & $.817^{* *}$ & $.812^{* *}$ & $.753^{* *}$ & & & \\
QGSY & 5.1724 & 1.21316 & $.776^{* *}$ & $.787^{* *}$ & $.801^{* *}$ & $.718^{* *}$ & $.824^{* *}$ & & \\
SMC & 5.2728 & 0.91897 & $.822^{* *}$ & $.851^{* *}$ & $.857^{* *}$ & $.817^{* *}$ & $.845^{* *}$ & $.864^{* *}$ & \\
\hline
\end{tabular}

**. 在. 01 水平 (双侧) 上显著相关

3.3 共同方法偏差检验

本研究借鉴周浩和龙立荣 [29]对共同方法偏差的检验方法，采用 Harman 单因素检验技 术进行相关的统计检验。对所有题项进行探索性因子分析, 查看未旋转时的因素分析, 主成 份分析法抽取了 4 个共同因子，得到第一个主成分所占的载荷量为 $28.845 \%$ ，低于 $40 \%$ 。所 以，各变量之间不存在严重的同源方法偏差问题，可以进行下一步的分析。

3.4 假设检验

本文主要采用 SPSS20.0 进行层级回归分析，先对所有变量做了标准化处理。分析结果 见表 3 。

由模型 2 可知: CN 对 RZSY 有显著正向影响（ $\beta=0.223, p<0.001)$ ，假设 H1a 成立; SP 对 RZSY 有显著正向影响（ $\beta=0.336, p<0.001)$ ，假设 H1b 成立; SJ 对 RZSY 有显著正向影 响（ $\beta=0.221, p<0.05)$, 假设 H1c 成立; GLD 对 RZSY 有显著正向影响（ $\beta=0.273$, $\mathrm{p}<0.001)$ ，假设 H1d 成立。

由模型 3 可知: SMC 正向调节 CN 与 RZSY 的关系 ( $\beta=0.166, p<0.05)$, 假设 H4a 成 立; SMC 正向调节 SP 与 RZSY 的关系 ( $\beta=0.222, p<0.05)$, 假设 H4b 成立; 但 SMC 对 SJ 与 RZSY 和 GLD 与 RZSY 的关系的调节未得到验证，假设 H4c 和 H4d 不成立。

由模型 5 可知: CN 对 QGSY 有显著正向影响 ( $\beta=0.370, p<0.001)$, 假设 H2a 成立; SP 对 QGSY 有显著正向影响（ $\beta=0.186, p<0.05)$ ，假设 H2b 成立; SJ 对 QGSY 有显著正向影响

$(\beta=0.286, p<0.01)$ ，假设 $H 2 c$ 成立; GLD 对 QGSY 有显著正向影响（ $\beta=0.223$, $\mathrm{p}<0.01)$ ，假设 $\mathrm{H} 2 \mathrm{~d}$ 成立。 
由模型 6 可知: SMC 正向调节 CN 与 QGSY 的关系 $(\beta=0.025, p<0.05)$, 假设 H5a 成 立; SMC 正向调节 SP 与 QGSY 的关系 ( $\beta=0.038, p<0.05)$, 假设 H5b 成立; SMC 正向调节 $\mathrm{SJ}$ 与 QGSY 的关系 $(\beta=0.025, \mathrm{p}<0.05)$ ，假设 H5c 成立; 但 SMC 对 GLD 与 QGSY 的关系的调 节未得到验证，假设 $\mathrm{H} 5 \mathrm{~d}$ 不成立。

表 3 回归分析结果 $(\mathrm{N}=294)$

\begin{tabular}{lllllll}
\hline \multirow{2}{*}{ 变量 } & \multicolumn{3}{c}{ 认知声誉 } & \multicolumn{3}{c}{ 情感声誉 } \\
\cline { 2 - 7 } & 模型 1 & 模型 2 & 模型 3 & 模型 4 & 模型 5 & 模型 6 \\
\hline 职位等级 & -0.008 & 0.093 & 0.101 & -0.053 & 0.029 & 0.046 \\
员工人数 & $0.297^{*}$ & $0.136^{* *}$ & $0.121^{*}$ & $0.159^{*}$ & 0.003 & -0.024 \\
生命周期 & 0.160 & -0.010 & -0.018 & $0.185^{*}$ & 0.011 & 0.041 \\
企业性质 & 0.003 & -0.009 & -0.029 & 0.036 & 0.030 & 0.000 \\
是否上市 & -0.640 & -0.065 & -0.038 & -0.456 & 0.152 & 0.139 \\
所属行业 & $0.044^{*}$ & 0.010 & 0.002 & $0.048^{* * *}$ & 0.012 & 0.006 \\
CN & & $0.223^{* * *}$ & -0.759 & & $0.370^{* * *}$ & 0.019 \\
SP & & $0.336^{* * *}$ & $1.399^{*}$ & & $0.186^{*}$ & -0.139 \\
SJ & & $0.221^{*}$ & -0.575 & & $0.286^{* *}$ & 0.358 \\
GLD & & $0.273^{* * *}$ & 0.376 & & $0.223^{* *}$ & 0.109 \\
SMC & & & 0.254 & & & $0.742^{* * * *}$ \\
SMC $* C N$ & & & $0.166^{*}$ & & & $0.025^{*}$ \\
SMC $*$ SP & & & $0.222^{*}$ & & & $0.038^{*}$ \\
SMC $* S J$ & & & -0.033 & & & $0.141^{*}$ \\
SMC $* G L D$ & & & -0.044 & & & -0.023 \\
R 方 & 0.243 & 0.780 & 0.793 & 0.147 & 0.714 & 0.772 \\
调整 R 方 & 0.227 & 0.771 & 0.782 & 0.129 & 0.704 & 0.759 \\
\hline
\end{tabular}

*表示 $\mathrm{p}<0.05 ， * *$ 表示 $\mathrm{p}<0.01 ， * * * \mathrm{p}<0.001$

\section{四、结果与讨论}

\section{1. 研究结果}

第一, 企业社会责任特征的四个维度分别正向影响企业的认知声誉和情感声誉。这一结 论填补了以往研究主要围绕企业社会责任的概念和内容展开讨论, 鲜有研究解释企业履行何 种形式的企业社会责任（即企业社会责任特征）会对企业声誉产生一定的影响, 以及对认知 声誉和情感声誉分别产生什么影响的理论空缺。此外, 企业的认知声誉对情感声誉有正向的 影响。这一结论也为 “认知和情感反应谁先产生” 再添一个新的论证。

第二，社交媒体能力正向调节企业社会责任特征（承诺、水平、时间选择）对情感声誉 的影响, 但对企业社会责任关联度和情感声誉关系的调节不显著; 社交媒体能力正向调节企 业社会责任特征 (承诺、水平) 对认知声誉的影响, 但对企业社会责任特征（时间选择、关 联度) 和认知声誉关系的调节不显著。其可能原因是: 通过社交媒体呈现出来的企业社会责 任活动, 利益相关者可能更多关注企业是否切实履行社会责任, 而对履行社会责任时表现的 行为是否与企业的业务范围相关并不关心, 尤其是在重大灾难性事件面前, 利益相关者只会 关注企业是否有实际行动以及履责的程度大小, 并不大会关心这个活动是否与企业的业务范 围相关。因此, 企业的社交媒体能力对企业社会责任关联度与企业声誉 (认知声誉和情感声 誉) 关系的正向调节呈现不显著。所谓时间选择, 也就是企业在履行社会责任时是主动积极 响应还是被动消极响应, 是基于内在本质还是迫于外在压力, 而这一本质通过社交媒体所呈 现出来, 直观上会增加或降低利益相关者对企业的好感度, 进而影响利益相关者对企业的情 感声誉评价, 但对企业的认知声誉评价并不仅仅受企业社会责任活动的影响, 还会受企业的 经营能力及实力等其他因素影响。也就是说并不能因为社交媒体所呈现的企业主动还是被动 履责而直接影响利益相关者对企业的认知声誉评价。因此, 企业的社交媒体能力对企业社会 
责任时间选择与认知声誉关系的正向调节呈现不显著。

\section{2. 管理启示}

第一，充分利用社交媒体平台。虽然本研究中社交媒体能力对企业社会责任特征和企业 双重声誉关系的正向调节并不都显著, 正因为如此, 管理层可以研究下企业应该如何充分利 用社交媒体平台, 将利益相关者所关注的内容呈现其上, 最大程度上发挥社交媒体的价值。

第二, 深入了解利益相关者的关注焦点。基于企业社会责任的内在本质, 多方调查研究 利益相关者更多关注的是哪些方面的内容, 进而确定是企业本身履责实力不够还是未通过多 方渠道将信息传递给利益相关者，找出原因，对症下药。

第三，提高企业的声誉管理水平。鉴于本研究得出的结论，认知声誉会正向影响情感声 誉, 因此管理层首先要做的还是提升企业的经营实力和影响力, 在此基础上多多展开企业的 社会责任活动, 而不要本末倒置。

\section{3. 研究局限}

本研究存在一定的局限性。主要表现在：第一，样本发放范围局限于浙江省，而浙江省 属于互联网发展较前卫的地域, 样本范围的局限性导致不得不考虑结论的普适性; 第二, 本 次问卷调查都是管理者对企业的自我评价, 会有一定的主观性, 接下来将探索是否有可能将 某企业管理层的自评和利益相关者的他评进行配对分析, 进一步验证研究结论并为企业管理 层提供具体可行的建议。

\section{Reference}

[1] Fombrun, C. J. (1996). Reputation: Realizing value from the corporate image. Cambridge, MA: Harvard Business Press.

[2] Walker, K. (2010). A systematic review of the corporate reputation literature: Definition, measurement, and theory. Corporate Reputation Review, 12(4), 357 - 387.

[3] Argyris, Y.A., and Ransbotham, S. (2016). "Knowledge entrepreneurship: Institutionalizing wikibased knowledge-management processes in competitive and hierarchical organizations." Journal of Information Technology 31(1), 226-239.

[4] Kane, G. (2015). "Enterprise social media: Current capabilities and future possibilities." MIS Quarterly Executive 14(1), 1-16.

[5] Ku, Y., Chen, R., and Zhang, H. (2013). "Why do users continue using social networking sites? An exploratory study of members in the United States and Taiwan.” Information \& Management 50(7), 571-581.

[6] Xu, J. (2016). "Retaining customers by utilizing technology-facilitated chat: Mitigating website anxiety and task complexity.” Information \& Management 53(5), 554-569.

[7] Mandviwalla, M., and Watson, R. (2014). "Generating capital from social media." MIS Quarterly Executive 13(2), 97-113.

[8] Melo T, Garrido-Morgado A. 2012. Corporate Reputation: A combination of social responsibility and industry. Corporate Social Responsibility and Environmental Management 19: 11 -31. https://doi.org/10.1002/csr.260

[9] Aguilera-Caracuel J, Guerrero-Villegas J. How Corporate Social Responsibility Helps MNEs to Improve their Reputation. The Moderating Effects of Geographical Diversification and Operating in Developing Regions[J]. Corporate Social Responsibility \& Environmental Management, 2017.

[10] Fieseler, C., and Fleck, M. (2013). "The pursuit of empowerment through social media: Structural social capital dynamics in CSR-blogging.” Journal of Business Ethics 118(4), 759-775.

[11] Lee, K., Oh, W., and Kim, N. (2013). "Social media for socially responsible firms: Analysis of Fortune 500's Twitter profiles and their CSR/CSIR ratings." Journal of Business Ethics 118(4), 791806.

[12] J Benitez, L Ruiz, J Llorens, A Castillo.(2017) Corporate social responsibility, employer reputation, and social media capability: an empirical investigation. Twenty-Fifth European Conference on Information Systems (ECIS), Guimarães, Portugal, 2017 
[13] Fombrun, C. J., \& Shanley, M. (1990). What's in a name: Reputation building and corporate strategy. Academy of Management Journal, 33(2), 233-258.

[14] Brammer S, Pavelin S. Building a Good Reputation[J]. European Management Journal, 2004, 22(6):704-713. [15] Lai C S, Chiu C J, Yang C F, et al. The Effects of Corporate Social Responsibility on Brand Performance: The Mediating Effect of Industrial Brand Equity and Corporate Reputation[J]. Journal of Business Ethics, 2010, 95(3):457-469.

[16] Maden C, Arıkan E, Telci E E, et al. Linking Corporate Social Responsibility to Corporate Reputation: A Study on Understanding Behavioral Consequences[J]. Procedia - Social and Behavioral Sciences, 2012, 58(7):655-664.

[17] Baraibardiez E, Sotorrío L L, Baraibardiez E, et al. The mediating effect of transparency in the relationship between corporate social responsibility and corporate reputation[J]. Rev.bras.gest.neg, 2018, 20(1):5-21.

[18] Shen ze. Research on the impact of CSR on corporate reputation from the perspective of consumers -comparative analysis of three industries [D]. Zhejiang university, 2006.

[19] Li haiqin, zhang zigang. An empirical study on CSR's influence on corporate reputation and customer loyalty [J]. Nankai management review,2010, 13(1):90-98. [20] Tian hong, jiang yufeng. An empirical study on the impact of social responsibility performance on corporate reputation -- the moderating effect of stakeholder pressure and moral decline [J]. Journal of social science, jilin university, 2015(2):71-79.

[21] Liu fengjun, li jingqiang, li hui. Empirical research on the relationship between corporate social responsibility characteristics and brand influence. China software science,2012,(1): 116-132.

[22] Manfred S. Components and Parameters of Corporate Reputation: An Empirical Study[J]. Schmalenbach Business Review,2004,56(1):46-72

[23] Benitez, J., Castillo, A., Llorens, J., and Braojos, J. (2016). "IT-enabled knowledge ambidexterity and innovation performance: The role of social media capability." In: Proceedings of the 22th Americas Conference on Information Systems. San Diego, California, USA, p.1-10.

[24] Braojos, J., Benitez, J., and Llorens, J. (2015a). "How do small firms learn to develop a social media competence?” International Journal of Information Management 35(4), 443-458.

[25] Fieseler, C., Fleck, M., and Meckel, M. (2010). "Corporate social responsibility in the blogosphere." Journal of Business Ethics 91(4), 599-614.

[26] Eberle, D., Berens, G., and Li, T. (2013). "The impact of interactive corporate social responsibility communication on corporate reputation.” Journal of Business Ethics 118(4), 731-746.

[27] Ennen E, Richter A. The whole is more than the sum of its parts- Or is it? A review of the empirical literature on complementarities in organizations.[J]. Social Science Electronic Publishing, 2010, 36(1):207-233.

[28] Xu Jinfa, Liu Liang. A Review of Corporate Reputation Definition and Measurement Research[J].Foreign Economy and Management, 2004, 26(9):25-30.

[29] Zhou Hao, long Lirong. Statistical test and control method of common method deviation [J]. Progress in Psychological Science, 2004 12: 942-950. 